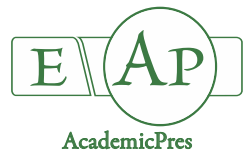

\title{
Roles of Some Plant Growth Regulators on Natural and Dark-Induced Senescence in Wheat Leaf Segments
}

\author{
Nihal GÖREN-SAĞLAM ${ }^{1 *}$, Fazilet Özlem ÇEKIÇ² \\ ${ }^{1}$ Istanbul University, Faculty of Science, Department of Biology, Istanbul, Turkey; gorenn@istanbul.edu.tr (*corresponding author) \\ ${ }^{2}$ Aksaray University, Faculty of Science and Letters, Department of Biology, Aksaray, Turkey; faziletozlem@gmail.com
}

\begin{abstract}
Senescence is an important developmental process in the life span of a plant. It is affected by several endogenous and exogenous factors such as hormones, darkness, and drought. Hormones are major endogenous factors that affect senescence intensively. In this study, our aim was to investigate the effects of different plant growth regulators; benzyl adenin (BA), spermine (Spm) and spermidine (Spd) on both natural and dark-induced senescence and on $\gamma$-aminobutyric acid (GABA) content in wheat leaf segments. Following the incubation, fresh and dry weight, chlorophyll and carotenoid contents; peroxidase activity, soluble protein, GABA and malondialdehyde (MDA) contents and cell death amount were determined. Our results demonstrate that Spm and Spd accelerated senescence under light and delayed it under dark conditions. BA has delayed leaf senescence under both conditions. Cell death and MDA content showed a correlation with the senescence process. GABA content was enhanced considerably by BA, Spm and Spd applications under light conditions. According to our results we can conclude that $\mathrm{Spm}$ and Spd could promote senescence on wheat segments under light conditions, whereas inhibit senescence under dark conditions. In addition, BA could retard the deleterious effects on senescence under both light and dark conditions.
\end{abstract}

Keywords: cell death; gamma-aminobutiric acid; plant growth regulators; polyamines; senescence; Triticum aestivum L.

One of the most important internal factors regulating leaf senescence is hormones (Ananieva et al., 2004). Hormones can be studied in two groups; hormones that stimulate senescence and hormones that delay senescence. Ethylene, abscisic acid (ABA), jasmonic acid (JA), and salicylic acid (SA) act as inducers of senescence, whereas cytokinins and gibberellins play important role in its suppression (Castillo and Leon, 2008). However, these effects may differ between plants. Benzyl Adenine (BA) is a plant hormone derived from cytokinin, which is known to delay senescence (Ananieva et al., 2004; Talla et al., 2016). Gören and Çağ (2007) have shown that the external application of BA delays senescence in sunflower cotyledons. The application of exogenous cytokinins also reduces the lipoxygenase activity and delays leaf senescence in the leaves of Uranium sativum L. (Liu and Huang, 2002). Pan et al. (2013) also indicated that 6-Benzylaminopurine application alleviated the effects of senescence by improving antioxidant activity and they suggested spraying of BA to Oryza sativa L. to increase the grain yield.

Polyamines are low molecular weight amines. Among polyamines Spm and Spd are found commonly as plant growth regulators in plants (Gill and Tuteja, 2010). Several studies show that externally applied Spm and Spd have a retarding effect on the process of senescence (Nambeesan $e t$ 
1231

al., 2010; Cai et al., 2015). Increasing the level of polyamines under stress conditions is known to be an adaptive strategy because of their roles in plant metabolism (Gupta et al., 2012). $\gamma$-aminobutyric acid (GABA) is a nonprotein amino acid and it can act as a signal molecule under stress conditions. GABA is synthesized from L-glutamate. GABA can also be occurred from the degradation of polyamine (PA) by the activity of polyamine oxidase (PAO) and diamine oxidase (DAO) (Shelp et al., 2012; Wang et al., 2014). The catabolism of spermine and spermidine feeds strongly the GABA pool (Fait et al., 2008). GABA shunt is also mentioned as a key signaling and metabolic pathway in senescence process (Ansari et al., 2014). Therefore, there is a strong correlation between GABA and senescence.

Dark-induced senescence has been a frequent subject of academic studies due to its rapid progression. It is still a matter of debate as to whether natural and dark-induced senescence are the same phenomenon. It has been found that there is a difference in gene expression between natural and dark-induced senescence in barley leaves, but it can be changed according to the plant species (BuchananWollaston et al., 2005).

Detached leaves are well suited for studying the effects of hormone application on senescence and studying various physiological and biochemical changes due to the senescence of chloroplasts and chlorophyll (Kleber-Janke and Krupinska, 1997). The aim of this study was to examine the effects of BA, Spm and Spd on natural and darkinduced senescence in detached wheat leaves by using some physiological analysis such as fresh and dry weight changes, pigment analysis, GABA and MDA contents, peroxidase activity and cell death amount.

\section{Materials and Methods}

Plant material, growth conditions and BA, Spd and Spm treatments

Wheat (Triticum aestivum L.) seeds were planted in moustrized perlit after surface sterilization with $10 \%$ commercial bleach and washed 5 times with sterile distilled water. They were grown in growth chamber $(16 \mathrm{~h}$ light, $8 \mathrm{~h}$ dark photoperiod and $\left.25 \pm 2{ }^{\circ} \mathrm{C}\right) .4$ of first leaf segments $(3$ $\mathrm{cm}$. each) from 10 days old wheat seedlings were placed in 5 $\mathrm{cm}$ diameter petri dishes containing $4 \mathrm{~mL}$ of BA (Sigma; B3408; plant cell culture)) (0.1 mM), Spm (Sigma; 85590; pure bases) (10 mM), and Spd (Sigma; S2626; pure bases) $(10 \mathrm{mM})$. The concentrations of BA, Spm and Spd were chosen according to Longo et al. (1978) (for BA); Cheng and Kao (1983) (for Spm and Spd) and our previous studies. Distilled water was used as control. After that, the first group was put into growth chamber for natural senescence and other group was covered with the aluminum foil and put in the growth chamber for dark-induced senescence. After 10 days all samples were used for analysis with 10 replicates.

\section{Fresh and dry weight analysis}

After the harvest, segments of wheat were weighed and placed in a BA, Spm and Spd solution. After 10 days, the segments were weighed and the fresh weight change was calculated. For dry weight analysis, the wheat segments were weighed after harvesting and placed in BA, Spm and Spd solutions. At the end of 10 days, the segments were weighed again and their dry weights were determined by keeping them in aluminum foil in $80{ }^{\circ} \mathrm{C}$ drying oven until their weight remained constant (3-4 days). It was analysed with 10 replicate tissue samples bulked 4 leaf segments.

\section{Analysis of pigment content}

Pigments were extracted by grinding the wheat segments in $90 \%$ ice-cold acetone with pestle and mortar and the total chlorophyll and carotenoid contents were determined spectrophotometrically (Shimadzu 1601) (Parsons and Strickland, 1963). It was analyzed with 10 replicate tissue samples bulked 4 leaf segments.

\section{Analysis of protein content}

The segment samples were homogenized with ice-cold $0.1 \mathrm{mmol} / \mathrm{L}$ sodium phosphate buffer ( $\mathrm{pH} 7.0)$. The homogenates were centrifuged at $13000 \mathrm{rpm}$ for $30 \mathrm{~min}$ at $4{ }^{\circ} \mathrm{C}$ and the supernatants were used for the determination of total soluble protein content and for the total peroxidase enzyme assay. The protein content of the extracts was determined according to Bradford (1976), using bovine serum albumin as a standard. It was analysed with 10 replicate tissue samples bulked 4 leaf segments.

\section{Peroxidase activity assay}

The reaction mixture consisted of guaiacol (volume fraction of $0.25 \%$ ) in $1 \mathrm{~mL}$ of $0.1 \mathrm{~mol} / \mathrm{L}$ sodium phosphate buffer, pH 7.0, containing $0.1 \%$ hydrogen peroxide. Crude enzyme extract $(60 \mu \mathrm{L})$ was added to initiate the reaction, which was measured spectrophotometrically at $470 \mathrm{~nm}$. The reaction is based on guaiacol oxidation and was recorded for $2 \mathrm{~min}$., and defined quantitatively as $\Delta \mathrm{A} / \mathrm{g} \cdot \mathrm{Fr} \cdot \mathrm{W} \cdot \mathrm{xMin}$ (Birecka et al., 1973). It was analyzed with 10 replicate tissue samples bulked 4 leaf segments.

\section{$G A B A$ assay}

GABA content was analyzed by HPLC (Agilent 1200) according to Bor et al. (2009). The samples were separated by a reversed-phase column (Supelco LC18; $250 \times 4.6 \mathrm{~mm}$, $5 \mu \mathrm{m})$ at $330 \mathrm{~nm}$ (DAAD dedector). 2hydroxynaphthaldehyde (HN) was used for the derivatization. The flow rate was $1 \mathrm{~mL} \mathrm{~min}{ }^{-1}$. The injection volume was $5 \mu \mathrm{L}$. methanol:water (62:38) was used as the mobile phase. The retention time of GABA was $12 \mathrm{~min}$. The content of GABA was calculated by comparing of the peak areas with the GABA standards.

\section{$M D A$ analysis}

MDA analysis was performed according to Karabal et al. (2003). $0.2 \mathrm{~g}$ of leaf samples were homogenized in $1 \mathrm{~mL}$ of trichloroacetic acid solution (TCA, 5\%) and centrifuged at $12,000 \mathrm{rpm}$ for $15 \mathrm{~min}$. The supernatant of the extract and thiobarbituric acid (TBA, $0.5 \%$ ) solution which is dissolved in TCA solution (20\%) were mixed and incubated for 25 min at $96^{\circ} \mathrm{C}$ in a water bath. After the incubation the tubes were cooled in an ice bath, and then they were centrifuged at $10,000 \mathrm{rpm}$ for 5 minutes. The supernatant was detected spectrophotometrically at 532 and $600 \mathrm{~nm}$. MDA content, the indicator of lipid peroxidation, was calculated by using extinction coefficient $\left(155 \mathrm{mM}^{-1} \mathrm{~cm}^{-1}\right)$. 
1232

\section{Measurement of cell death}

Cell death was measured spectrophotometrically by Evans blue staining of detached leaves (Guo and Crawford, 2005). Detached leaves that submerged in a $0.1 \%(\mathrm{w} / \mathrm{v})$ aqueous solution of Evans blue dye (Sigma-Aldrich). They were subjected to two 5-min cycles of vacuum followed by 30 min under vacuum. The leaves were then washed three times with distilled water $(15 \mathrm{~min}$ each). Dye bound to dead cells was solubilized in 50\% (v/v) methanol and $1 \%(\mathrm{w} / \mathrm{v})$ SDS at $60^{\circ} \mathrm{C}$ for $30 \mathrm{~min}$ and then quantified by absorbance at $600 \mathrm{~nm}$. For $100 \%$ cell death, the detached leaves were heated at $100^{\circ} \mathrm{C}$ for $5 \mathrm{~min}$ before staining. Four leaves were pooled for each sample. 10 samples were analyzed and this experiment was repeated three times with equivalent results.

\section{Statistical analysis}

Each treatment was analyzed with 10 replicate. The data presented here are the mean values \pm SE of 10 independent experiments.

\section{Results}

To investigate the effects of BA, Spm and Spd on natural and dark-induced senescence, detached leaves were placed in petri dishes containing distilled water (as control) and BA, Spm and Spd solutions. We found that BA inhibited the senescence process in both dark and light according to the control group, while Spm and Spd increased the senescence in the light and stimulated it in the dark (Fig. 1).

\section{Fresh and dry weight results}

The amounts of fresh weight, dry weight and the water loss percentage observed in wheat segments soaked in BA, Spm and Spd solutions are given in Fig. 2, Fig. 3 and Fig. 4, respectively. Fresh weight was calculated by subtracting the final weights of the segments recorded after being soaked in BA, Spm and Spd solutions from their initial weights recorded before soaking in the solutions. As seen in Fig. 2, the highest number of fresh weight change takes place in Spm and Spd applications. Interestingly, it has also been determined that light or darkness does not make much difference.

When dry weight amounts are examined, it is seen that light/BA and light/control groups have the highest amount of dry weight (Fig. 3), while a reduction of the dry weight has been observed in other groups. A calculation of the percentage of water loss has shown that the least amount of water loss (70\%) occurs in the light/BA group (Fig. 4), whereas the highest amount of water loss $(90 \%)$ has been observed in the darkness/control group.

\section{Pigment content}

Following applications on cut wheat segments, it has been confirmed through observations and the chlorophyll content that with regards to segments kept in the light and the dark, BA delays yellowing, and eventually the senescence process, more in comparison to the control group. On the other hand, Spm and Spd applications have revealed that light promotes senescence, while under darkness the application of Spm and Spd delayed the senescence when compared to the control group (Fig. 1; Fig. 5).

In our study we found a significant reduction in carotenoid contents by Spm and Spd application under light conditions. The groups with the highest amount of carotenoids are light/BA and dark/BA groups (Fig. 6).

\section{Soluble protein amounts}

With regards to the promotion of the senescence process, a decrease was observed in the protein amounts (Fig. 7).

\section{Peroxidase activity}

According to the results obtained, peroxidase activity was low in BA applications, in both and light conditions, whereas Spm and Spd applications showed high levels of peroxidase activity in the light and low levels of peroxidase activity in dark (Fig. 8).

\section{Lipidperoxidation}

Malondialdehyde (MDA) is an indicator of lipid peroxidation. In our study we found that Spm and Spd applications increased MDA content significantly under light conditions when compared to the control group. MDA content of Spm application was found 2.21 fold higher than that of control plants, whereas Spd application increased MDA content 3.42 fold as compared to the control group. However; under dark conditions we did not find an increase in all of the applications as compared to their control plants (Fig. 9).

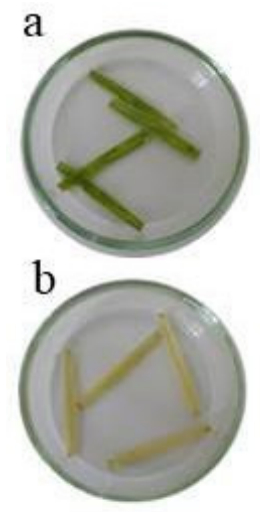

Control
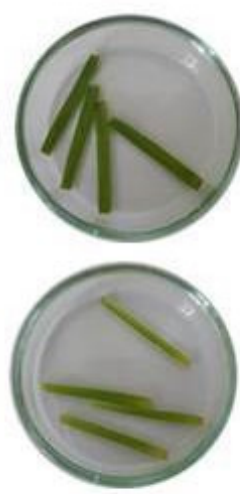

BA

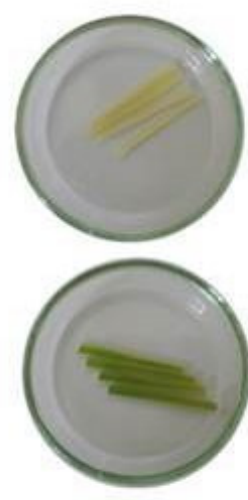

Spm

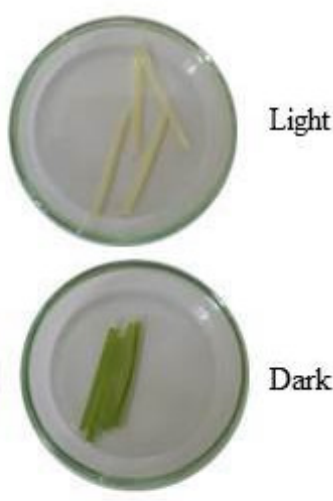

Spd

Fig. 1. Wheat leaf segments after incubation in distilled water (control), BA, Spm and Spd, respectively. (a) light (b) dark 


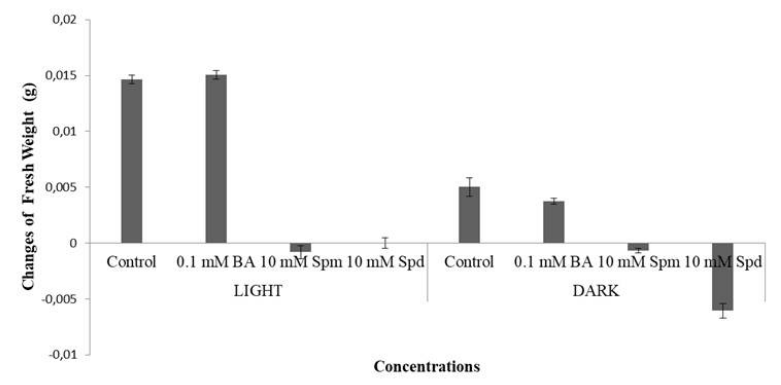

Fig. 2. Comparisons of fresh weight after incubation in distilled water (control), BA, Spm and Spd under light and dark conditions. Values are means \pm S.E.

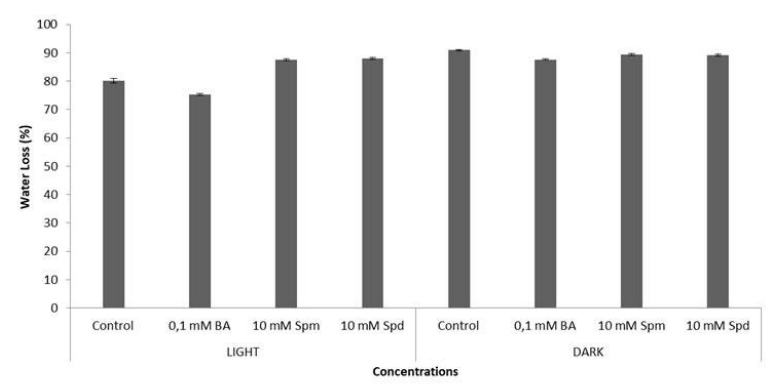

Fig. 4. Comparisons of water loss (\%) after incubation in BA, Spm and Spd under light and dark conditions. Values are means \pm S.E.

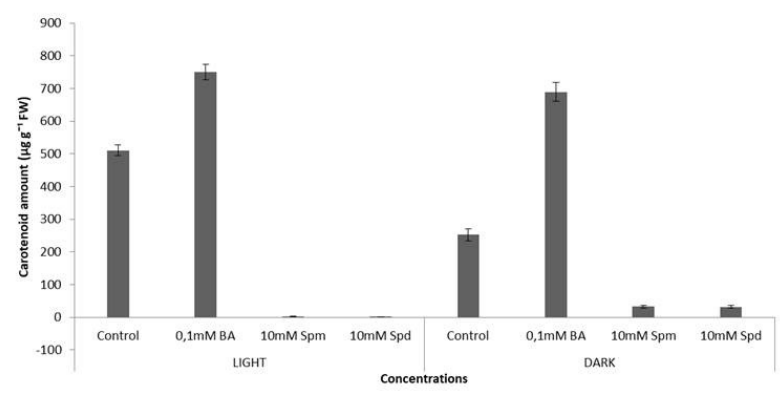

Fig. 6. Effects of BA, Spm and Spd treatments on carotenoid content in wheat leaf segments under light and dark conditions. Values are means \pm S.E.

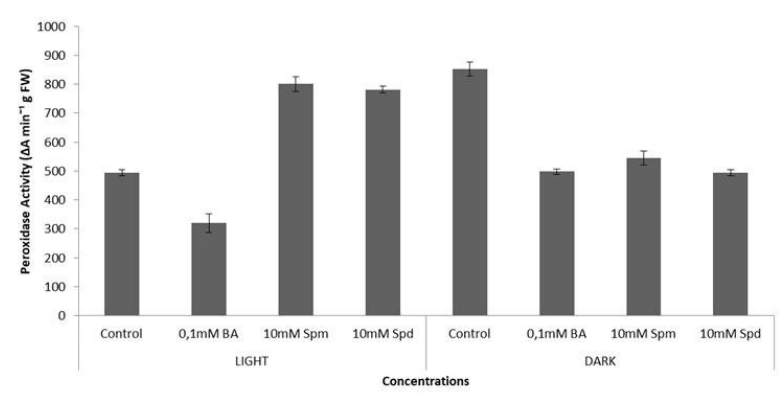

Fig. 8. Effects of BA, Spm and Spd treatment on peroxidase activity under light and dark conditions. Values are means \pm S.E.

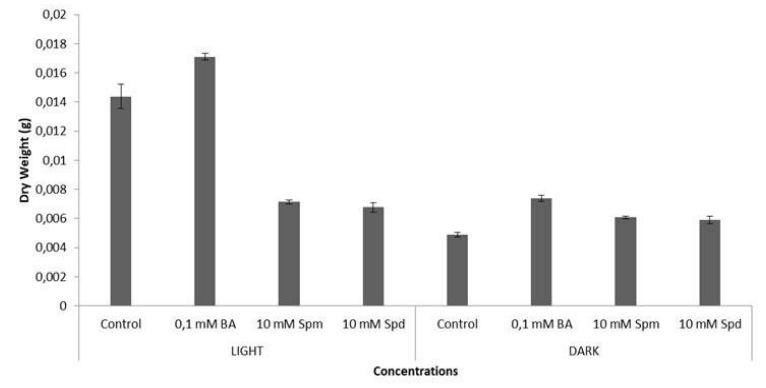

Fig. 3. Comparisons of dry weight after incubation in BA, Spm and Spd under light and dark conditions. Values are means \pm S.E.

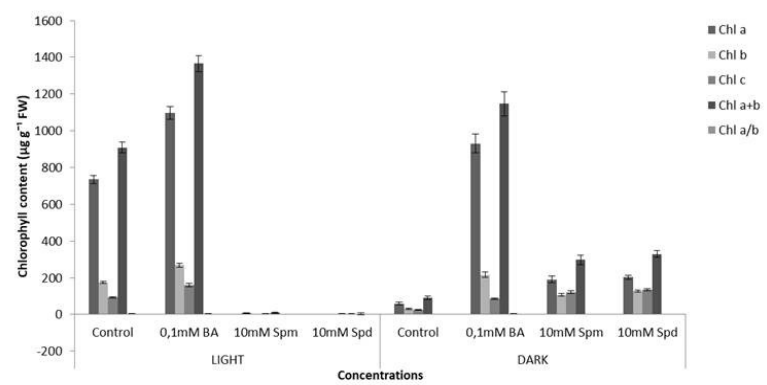

Fig. 5. Effects of BA, Spm and Spd treatments on chlorophyll content in wheat leaf segments under light and dark conditions. Values are means \pm S.E.

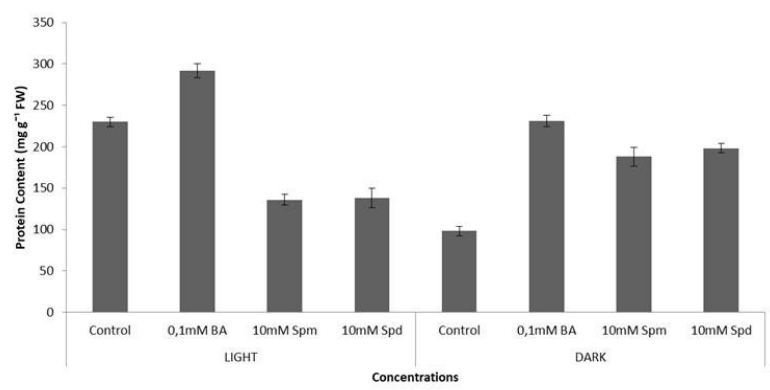

Fig. 7. Soluble protein contents of the wheat leaf segments after incubation in BA, Spm and Spd under light and dark conditions. Values are means \pm S.E.

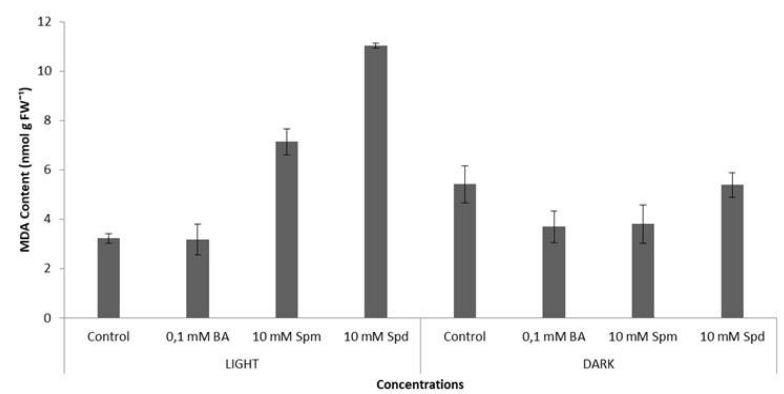

Fig. 9. Effects of BA, Spm and Spd treatment on MDA content under light and dark conditions. Values are means \pm S.E. 
1234

\section{Cell death}

Cell death indicated as loss of plasma membrane integrity. Our results showed that Spm and Spd applications increased cell death (\%) compared to control plants under light conditions (Fig. 10).

Spd application showed the highest increase at 3,6 fold higher than the control plants. Under dark conditions, Spd application was found 1.7 fold higher than control group.

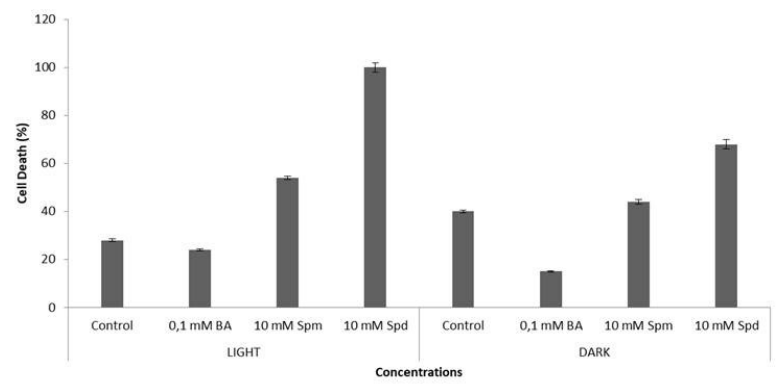

Fig. 10. Effects of BA, Spm and Spd treatment on cell death amount under light and dark conditions. Values are means \pm S.E.

\section{Discussion}

Senescence is the last stage in plant development that culminates in the death of cells, tissues, organs or the end of a life cycle (Lim et al., 2007; Liu et al., 2008). In terms of the transport and recycling of important nutrients such as nitrogen, sulfur, phosphorus and potassium, senescence is vital for plant development (Hörtensteiner and Feller, 2002; Balazadeh et al., 2008a).

\section{$B A$ delayed senescence under light and dark conditions}

Several researchers have reported that senescence could also occur in healthy plants that grow in the most favorable growth conditions (Srivastava, 2002), as well as in adverse environmental conditions (drought, high temperatures, lack of nitrogen, insufficient light and disease) ( $\mathrm{He}$ and Gan, 2002). In light of studies on the process of senescence, its effects on yellowing have been examined through a combined application of hormones, which constitute the internal factors, and changes to light intensity regarded as an external factor. Our study reports that BA, a cytokinin derivative, delays the senescence process in dark and light according to the controls applied, while Spm and Spd stimulate the process in the light and delay it in the darkness (Fig. 1). We are in agreement with previous studies regarding the effects of Spd and Spm on the senescence process (Pandey et al., 2000). Gören and Çă̆ (2007) have shown that the external application of benzyl adenine delays senescence in sunflower cotyledons. The application of exogenous cytokines also reduces the lipoxygenase activity and delays the leaf senescence in the leaves of Uranium sativum L. (Liu and Huang, 2002). These results confirm the inhibitory role of cytokinins in the leaf senescence.

\section{GABA content}

In our study under light conditions we found a significant increase in GABA content by BA (3.05 fold), Spm (2.29 fold) and Spd (2.02 fold) applications; however, we did not find an increase in GABA content under dark conditions as compared to control group (Fig. 11).

In our study, we found that GABA content was enhanced by BA, Spm and Spd treatments under light conditions when compared to control group.

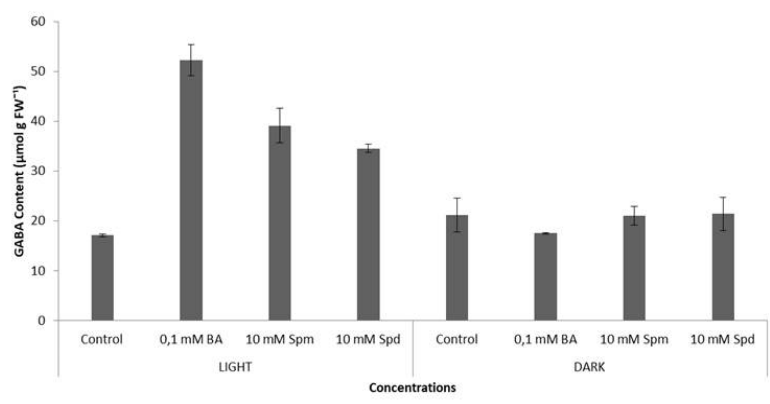

Fig. 11. Effects of BA, Spm and Spd treatment on GABA content under light and dark conditions. Values are means \pm S.E.

Fresh and dry weight were changed under light and dark conditions

The amounts of fresh weight, dry weight and the water loss in wheat segments soaked with BA, Spm and Spd solutions are given in Figs. 2, 3, 4, respectively. As seen in Fig. 2, the highest number of fresh weight change takes place in Spm and Spd applications. Interestingly, we found that light versus dark does not make much difference.

When dry weight amounts are examined, it is seen that light/BA and light/control groups have the highest amount of dry weight (Fig. 3), while a reduction of the dry weight has been observed in other groups.

\section{$B A$ prevented water loss under light conditions}

A calculation of the percentage of water loss shows that the least amount of water loss (70\%) occurrs in light/BA group (Fig. 4), whereas the highest amount of water loss (90\%) was observed in the control group under darkness.

\section{$B A$ delayed yellowing, while Spm and Spd promoted} senescence under light

During the senescence process, leaf cells are exposed to regular changes in structure, metabolism and gene expression (Lim et al., 2007). According to our observations and chlorophyll content results, BA delayed yellowing under light and dark conditions, and eventually the senescence process, as compared to the control group. On the other hand, Spm and Spd applications revealed light promoted senescence, while darkness delayed when compared to the control group (Fig. 1; Fig. 5). Our results correlate with the existing literature as well. Following the application of cytokines, a leaf that has gone completely yellow may promote refoliation, and reorganize the protein synthesis and photosynthetic activity (Venkatarayappa et al., 1984). 
In our study, we found a significant reduction in carotenoids under Spm and Spd applications. The groups with the highest amount of carotenoids are light/BA and dark/BA groups (Fig. 6).

Visible changes in the leaf colour characterized by a color switch from green to yellow take place during the final stages of the senescence following loss of chlorophyll and degradation of the structure of the chloroplasts (Balazadeh et al., 2008a). The first and most obvious change in cell structure is the degradation of chloroplasts containing about $70 \%$ of the leaf proteins. Biochemically, senescence is characterized by the degradation of macro-molecules, such as chlorophylls, proteins, membrane lipids and RNA, and metabolically these events replace the carbon assimilation (Balazadeh et al., 2008b). In our study we found that the changes in protein content are parallel to changes in the senescence process following the BA, Spm and Spd application. With regards to the promotion of the senescence process, a decrease has been observed in the protein amounts (Fig. 7). These results correlate with the literature cited above.

Increased peroxidase activity is an important indicator of senescence (Sağlam-Çağ, 2007). According to the results obtained, peroxidase activity is low in BA applications both in the light and dark, whereas Spm and Spd applications show high levels of peroxidase activity in the light and low levels of peroxidase activity in dark conditions (Fig. 8).

GABA was enhanced by BA, Spm and Spd under light conditions

Amino acid content can increase under stress conditions (Patterson et al., 2009). Increased levels of putrescine and GABA levels under salt stress may represent as indicators of senescence and/or cell damage. Therefore; putrescine and GABA have been mentioned as potential senescence markers (Patterson et al., 2009). Previous studies have reported the fluctuations in the GABA content along the day and during senescence (Ansari et al., 2005). In our study, we found that GABA content was enhanced by Spm and Spd treatments under light conditions. This increase could be due to the senescence process under light conditions.

Foliar application of benyzladenine and putrescine were able to increase drought tolerance in wheat plants (Gupta et al., 2012). Roberts (2007) demonstrated that application of benzyl adenine could affect genes related with GABA in Arabidopsis seedlings. GABA can also be regulated by hormones such as cytokinins (Roberts, 2007). Similar to previous studies, we found that under light conditions, BA treatment increased GABA content as compared to control plants. This increase could help to tolerate the detrimental effects of senescence and GABA can act as a signal molecule under these conditions. In another study, it was reported that the activities of GDH and GAD were induced by senescence and also different stresses (Ansari et al., 2005; Forde and Lea, 2007). Ansari et al. (2014) indicated that the signal role of GABA could be a tremendous potential in the field of agricultural crop productivity in senescence process. Therefore; the role of GABA in senescence process should be well identified.

PA has important roles in maintaining of ion balance, membrane stabilization, and anti-senescence (Guo et al., 2012). Several studies have indicated the close relationship between polyamines and GABA. GABA can be occurred via polyamine degradation pathway (Yang et al., 2013; Wang et al., 2014). Exogenous gamma-aminobutyric acid modulates the biosynthesis and degradation of polyamine metabolism (Wang et al., 2014). In a previous study it was reported that under hypoxia stress exogenous GABA caused an increase in the leaf GABA content and also arginine, methionine and ornithine contents which induced polyamines contents (Long-Quan et al., 2012). Exogenous GABA application could also alleviate the stress injury by inducing PA biosynthesis and it could help to reduce PA degradation in muskmelon plants ( $\mathrm{Hu}$ et al., 2015). Therefore; in our study the increase in GABA content under Spd and Spm applications could also be due to the role of PA on GABA pathway. And there could be a strong relationship between PA and GABA during senescence process.

GABA has also an important role in protecting the structure of chloroplasts and especially PSII under stress conditions (Xiang et al., 2016). In our study, BA treatment under light conditions caused an increase in GABA content and this increase could lead to protect the carotenoid content.

\section{Lipid peroxidation was increased by senescence}

Malondialdehyde (MDA) is an indicator of lipid peroxidation. In our study we found that Spm and Spd applications increased MDA content significantly under light conditions when compared to the control group. This increase could be due to the senescence in the light. Cell death is an important marker for the loss of plasma membrane integrity, like MDA. Our cell death results are also parallel with our MDA results indicating membrane integrity during the senescence process.

\section{Conclusions}

In conclusion, this study has demonstrated that the effect of BA, Spm and Spd on the senescence of wheat leaf segments under light and dark conditions. The data presented in this study show that Spm and Spd can promote senescence on segments under light conditions, whereas inhibit it under dark conditions as compared to the control group. GABA content can be enhanced under light conditions by BA, Spm and Spd applications. In addition, BA could retard the deleterious effects on senescence both light and dark conditions.

\section{Acknowledgements}

This study was supported by the Research Fund of Istanbul University (project numbers: 40796 and 54469). We are grateful to Dr. Lütfü Demir from Mısır Araştırma Enstitüsü, Adapazarı for providing the wheat seeds.

\section{Conflict of Interest}

The authors declare that there are no conflicts of interest related to this article. 
1236

\section{References}

Ananieva K, Malbeck J, Kamínek M, Van Staden J (2004). Changes in endogenous cytokinin levels in cotyledons of Cucurbita pepo (zucchini) during natural and dark-induced senescence. Physiologia Plantarum 122(1):133-142.

Ansari MI, Lee RH, Chen SCG (2005). A novel senescence-associated gene encoding $\gamma$-aminobutyric acid (GABA):pyruvate transaminase is upregulated during riceleaf senescence. Physiologia Plantarum 123(1):18.

Ansari MI, Hasan S, Jalil SU (2014). Leaf senescence and GABA shunt. Bioinformation 10(12):730-732.

Balazadeh S, Parlitz S, Mueller-Roeber B, Meyer RC (2008a). Natural developmental variations in leaf and plant senescence in Arabidopsis thaliana. Plant Biology 10:136-147.

Balazadeh S, Riaño-Pachón DM, Mueller-Roeber B (2008b). Transcription factors regulating leaf senescence in Arabidopsis thaliana. Plant Biology 10:63-75.

Birecka H, Briber KA, Catalfamo JL (1973). Comparative studies on tobacco pith and sweet potato root isoperoxidases in relation to injury, indoleacetic acid, and ethylene effects. Plant Physiology 52(1):43-49.

Bor M, Seckin B, Ozgur R, Yllmaz O, Ozdemir F, Turkan I (2009). Comparative effects of drought, salt, heavy metal and heat stresses on gamma-aminobutryric acid levels of sesame (Sesamum indicum L.). Acta Physiologiae Plantarum 31(3):655-659.

Bradford MM (1976). A rapid and sensitive method for the quantitation of microgram quantities of protein utilizing the principle of protein-dye binding. Analytical Biochemistry 72(1-2): 248-254.

Buchanan-Wollaston V, Page T, Harrison E, Breeze E, Lim PO, Nam HG, Leaver CJ (2005). Comparative transcriptome analysis reveals significant differences in gene expression and signalling pathways between developmental and dark/starvation-induced senescence in Arabidopsis. The Plant Journal 42(4):567-585.

Cai G, Sobieszczuk-Nowicka E, Aloisi I, Fattorini L, Serafini-Fracassini D, Del Duca $S$ (2015). Polyamines are common players in different facets of plant programmed cell death. Amino Acids 47(1):27-44.

Castillo MC, Leon J (2008). Expression of the $\beta$-oxidation gene 3-ketoacylCoA thiolase 2 (KAT2) is required for the timely onset of natural and dark-induced leaf senescence in Arabidopsis. Journal of Experimental Botany 59(8):2171-2179.

Cheng SH, Kao CH (1983). Localized effect of polyamines on chlorophyll loss. Plant and Cell Physiology 24(8):1463-1467.

Fait A, Fromm H, Walter D, Galili G, Fernie AR (2008). Highway or byway: the metabolic role of the GABA shunt in plants. Trends in Plants Science 13(1):1419.

Forde BG, Lea PJ (2007). Glutamate in plants: metabolism, regulation, and signalling.Journal of Experimental Botany 58(9):2339-2358.

Gill SS, Tuteja N (2010). Polyamines and abiotic stress tolerance in plants. Plant Signalingand Behavior 5(1):26-33.

Gören N, Çağ S (2007). The effect of indole-3-acetic acid and benzyladenine on sequential leaf senescence on Helianthus annuus L. seedlings. Biotechnology \& Biotechnological Equipment 21(3):322-327.

Guo FQ, Crawford NM (2005). Arabidopsis nitric oxide synthasel is targeted to mitochondria and protects against oxidative damage and dark-induced senescence. The Plant Cell 17(12):3436-3450.

Guo Y, Yang R, Chen H, Song Y, Gu Z (2012). Accumulation of $\gamma$ aminobutyric acid in germinated soybean (Glycine max $\mathrm{L}$.) in relation to glutamate decarboxylase and diamine oxidase activity induced by additives under hypoxia. European Food Research and Technology 234(4):679-687.

Gupta S, Agarwal VP, Gupta NK (2012). Efficacy of putrescine and benzyladenine on photosynthesis and productivity in relation to drought tolerance in wheat (Triticum aestivum L.). Physiology and Molecular Biology of Plants 18(4):331-336.

He Y, Gan S (2002). A gene encoding an acyl hydrolase is involved in leat senescence in Arabidopsis. The Plant Cell 14(4):805-815.

Hörtensteiner S, Feller U (2002). Nitrogen metabolism and remobilization during senescence. Journal of Experimental Botany 53(370):927-937.

Hu X, Xu Z, Xu W, Li J, Zhao N, Zhou Y (2015). Application of $\gamma$ aminobutyric acid demonstrates a protective role of polyamine and GABA metabolism in muskmelon seedlings under $\mathrm{Ca}(\mathrm{NO} 3)_{2}$ stress. Plant Physiology and Biochemistry 92:1-10.

Karabal E, Yücel M, Öktem HA (2003). Antioxidant responses of tolerant and sensitive barley cultivars to boron toxicity. Plant Science 164(6):925933.

Kleber-Janke T, Krupinska K (1997). Isolation of cDNA clones for genes showing enhanced expression in barley leaves during dark-induced senescence as well as during senescence under field conditions. Planta 203(3):332-340.

Lim PO, Kim HJ, Nam HG (2007). Leaf senescence. Annual Reviews on Plant Biology 58:115-136.

Liu L, Zhou Y, Zhou G, Ye R, Zhao L, Li X, Lin Y (2008). Identification of early senescence-associated genes in rice flag leaves. Plant Molecular Biology 67(1-2):37-55.

Liu X, HuangB (2002). Cytokinin effects on creeping bentgrass response to heat stress. CropScience 42(2):466-472.

Long-Quan F, Li-wen Y, Hong-bo G, Xiao-lei W, Qing-ping X, Bin-Bin G (2012). Effects of exogenous $\gamma$-aminobutyric acid on polyamine metabolism of melon seedlings under hypoxia stress. The Journal of Applied Ecology 23:1599-1606.

Longo GP, Olginati M, Rossi G, Valente M, Longo CP (1978). Effect of brief treatments with benzyladenine on growth and development of watermelon cotyledons. Plant, Cell and Environment 1(1):39-43.

Nambeesan S, Datsenka T, Ferruzzi MG, Malladi A, Mattoo AK, Handa AK (2010). Overexpression of yeast spermidine synthase impacts ripening, senescence and decay symptoms in tomato. The Plant Journal 63(5):836-847.

Noodén LD (1988). Abscisic acid, auxin and other regulators of senescence. In: Noodén LD, Leopold AC (Eds). Senescence and aging in plants. Academic Press, San Diego, CA, pp 1-50.

Pan S, Rasul F, Li W, Tian H, Mo Z, Duan M, Tang X (2013). Roles of plant growth regulators on yield, grain qualities and antioxidant enzyme activities in super hybrid rice (Oryza sativa L.). Rice 6(1):9-13.

Pandey S, Ranade SA, Nagar PK, Kumar N (2000). Role of polyamines and ethylene as modulators of plant senescence. Journal of Bioscience 25(3):291-299. 
Parsons TR, Strickland JDH (1963). Discussion of spectrophotometric determination of marine plant pigments, with revised equation for ascertaining chlorophylls and carotenoids. Journal of Marine Research 21:155-163.

Patterson JH, Newbigin ED, Tester M, Bacic A, Roessner U (2009). Metabolic responses to salt stress of barley (Hordeum vulgare L.) cultivars, 'Sahara' and 'Clipper', which differ in salinity tolerance. Journal ofExperimental Botany 60(14):4089-4103.

Roberts MR (2007). Does GABA act as a signal in plants? Plant Signaling and Behavior 2:408-409.

Sağlam-Çağ $S$ (2007). The effect of epibrassinolide on senescence in wheat leaves. Biotechnology and Biotechnological Equipment 21(1):63-65.

Shelp BJ, Mullen RT, Waller JC (2012). Compartmentation of GABA metabolism raises intriguing questions. Trends in Plant Science 17(2):57-59.

Smart CM (1994). Gene expression duringleaf senescence. New Phytologist 126(3):419-448.

Sobieszczuk-Nowicka E, Zmienko A, Samelak-Czajka A, Łuczak M, Pietrowska-Borek M, Iorio R, Legocka J (2015). Dark-induced senescence of barley leaves involves activation of plastid transglutaminases. Amino Acids 47(4):825-838.

Srivastava LM (2002). Plant growth and development: hormones and environment. Academic Press, California.
Talla SK, Panigrahy M, Kappara S, Nirosha P, Neelamraju S, Ramanan R (2016). Cytokinin delays dark-induced senescence in rice by maintaining the chlorophyll cycle and photosynthetic complexes. Journal of Experimental Botany 67(6):1839-1851.

Venkatarayappa T, Fletcher RA, Thompson JE (1984). Retardation and reversal of senescence in bean leaves by benzyladenine and decapitation. Plant and Cell Physiology 25(3):407-418.

Wang C, Fan L, Gao H, Wu X, Li J, Lv G, Gong B (2014). Polyamine biosynthesis and degradation are modulated by exogenous gammaaminobutyric acid in root-zone hypoxia-stressed melon roots. Plant Physiology and Biochemistry 82:17-26.

Woo HR, Kim JH, Kim J, Kim J, Lee U, Song IJ, Lim, PO (2010). The RAV1 transcription factor positively regulates leaf senescence in Arabidopsis.Journal of Experimental Botany 61(14):3947-3957.

Xiang L, Hu L, Xu W, Zhen A, Zhang L, Hu X (2016). Exogenous $\gamma$ aminobutyric acid improves the structure and function of photosystem II in muskmelon seedlings exposed to salinity-alkalinity stress. PloS One 11(10):e0164847.

Yang R, Guo Q, Gu Z (2013). GABA shunt and polyamine degradation pathway on $\gamma$-aminobutyric acid accumulation in germinating fava bean (Viciafaba L.) under hypoxia. Food Chemistry 136(1):152-159.

Zhang H, Zhou C (2013). Signal transduction in leaf senescence. Plant Molecular Biology 82(6):539-545. 